\title{
SYNCHRONIZATION VIA INTERACTING REINFORCEMENT
}

\author{
PAOLO DAI PRA, * Università degli Studi di Padova \\ PIERRE-YVES LOUIS, ${ }^{* *}$ Université de Poitiers \\ IDA G. MINELLI, ${ }^{* * *}$ Università degli Studi dell'Aquila
}

\begin{abstract}
We consider a system of urns of Pólya type, containing balls of two colors; the reinforcement of each urn depends on both the content of the urn and the average content of all urns. We show that the urns synchronize almost surely, in the sense that the fraction of balls of a given color converges almost surely as time tends to $\infty$ to the same limit for all urns. A normal approximation for a large number of urns is also obtained.
\end{abstract}

Keywords: Interacting system; synchronization; urn model

2010 Mathematics Subject Classification: Primary 60K35; 82C31

\section{Introduction}

Synchronization phenomena for stochastic systems have received a considerable interest in the last decade, both for their impact in applications and their intrinsic mathematical beauty. We make no attempt to give an overview of the many lines of research related to synchronization; we rather mention only two of them, which are related but developed mostly independently.

The word synchronization is often referred to as phase synchronization of pulsating systems. Interesting examples are provided by cellular dynamics. The concentration of certain molecules within cells vary over time due to chemical reactions, and may lead to periodic behavior. Chemical reactions may also involve molecules in the extracellular space, producing interactions between different cells: phase synchronization is actually observed when the interaction is sufficiently strong (see [13] for an account and a remarkable application). For this and other applications, a stochastic model that reasonably approximates the dynamics of phases is a noisy version of the Kuramoto model (see [1] and [6]). It is a system of $N$ coupled random rotators that, for a sufficiently small coupling parameter, show decoherence of phases: if $\theta_{j}(t)$ denotes the phase of the $j$ th oscillator at time $t$ then the distribution of

$$
\rho:=\left|\frac{1}{N} \sum_{j=1}^{N} \mathrm{e}^{\mathrm{i} \theta_{j}(t)}\right|
$$

is, for large $N$ and large $t$, concentrated near 0 . Such decoherence is lost as the coupling parameter is increased beyond a critical value.

\footnotetext{
Received 10 January 2013; revision received 8 July 2013.

* Postal address: Dipartimento di Matematica, Università degli Studi di Padova, via Trieste 63, I-35121 Padova, Italy. Email address: daipra@math.unipd.it

** Postal address: Laboratoire de Mathématiques et Applications, UMR 7348 CNRS, Université de Poitiers, 11 Boulevard Marie et Pierre Curie, 86962 Technopole du Futuroscope, Chasseneuil Cedex, France.

Email address: pierre.yves.louis@univ-poitiers.fr

*** Postal address: Dipartimento di Ingegneria e Scienze dell'Informazione e Matematica, Università degli Studi dell'Aquila, Via Vetoio (Coppito 1), 67100 Coppito (AQ), Italy. Email address: ida.minelli@dm.univaq.it
} 
In other contexts, the notion of synchronization is used even in the absence of an underlying periodic motion. In some models for metastable behavior (see, e.g. [2] and [3]), one considers a system of many particles subject to a symmetric, double-well potential, and interacting through an attractive potential that, in the models that are more mathematically treatable, is assumed to be of mean-field type. At sufficiently low temperatures, the majority of particles are found in the same well, thus breaking the symmetry of the model. Synchronization, in this context, is this coherent behavior of the majority.

In this paper we also consider a system whose components are subject to an attractive, mean-field interaction. We however add an ingredient to the model: reinforcement. Generally speaking, by reinforcement in a stochastic dynamic we mean any mechanism for which the probability that a given transition occurs has an increasing dependence on the number of times that 'similar' transitions have taken place in the past. The most elementary model of reinforcement is the Pólya urn.

The simplest Pólya urn model consists of an urn which contains balls of two different colors (for example, at time $t=0, b$ white and $a$ red balls). At each discrete time a ball is drawn and it is replaced in the urn together with another ball of the same color.

Let $Z_{t}$ be the fraction of red balls at time $t$, namely, the conditional probability of drawing a red ball at time $t+1$, given the fraction of red balls at time $t$. A well-known result (see, for instance, [5]) says that $Z_{t}$ is a bounded martingale and, in particular, $\lim _{t \rightarrow \infty} Z_{t}=Z_{\infty}$ almost surely (a.s.), where $Z_{\infty}$ has a beta distribution with parameters $a$ and $b$.

Here we present a modified model in which we consider a set of $N$ Pólya urns and we introduce a 'mean field interaction' among them:

- at time 0 , each urn contains $a$ red and $b$ white balls, $a \geq 1$ and $b \geq 1$;

- at each time $t+1$, given the fraction $Z_{t}(j)$ for $j=1, \ldots, N$ of red balls in each urn $j$ at time $t$, independently of what happens in all the other urns, a new red ball is replaced in urn $i$ with conditional probability $\alpha Z_{t}+(1-\alpha) Z_{t}(i)$, where $Z_{t}$ is the total fraction of red balls at time $t$, i.e. $Z_{t}=(1 / N) \sum_{i=1}^{N} Z_{t}(i)$ and $\alpha \in[0,1]$.

The case $\alpha=0$ corresponds to $N$ independent copies of the classical Pólya urn described above. Thus, for $i=1,2, \ldots, N$, the $Z_{t}(i)$ converge as $t \rightarrow+\infty$ to independent and identically distributed (i.i.d.) random variables, whose distribution is beta with parameters $a$ and $b$. We show that, for $\alpha>0, Z_{i}(t)$ is no longer a martingale; however, its almost-sure limit as $t \rightarrow+\infty$ exists, and it is the same for all $i=1,2, \ldots, N$. We refer to this phenomenon as almost-sure synchronization. We also obtain bounds on the $L^{2}$ distance between $Z_{i}(t)$ and $Z_{j}(t), i \neq j$, which tends to 0 uniformly in $N$ and as a power law in $t$.

We note that more general and complex models of interacting Pólya urns have been considered recently by various authors (see, e.g. [4], [9], [11], and [12]). In particular, urns with mean-field interaction have been considered in [7] and [8], but with a different reinforcement scheme: the probability of drawing a red (or black) ball is proportional to the exponential of the number of red balls, rather than to the number of red balls, leading to a quite different synchronization picture.

The paper is organized as follows. In Section 2 we give some basic properties of the model, while Section 3 is devoted to synchronization. Finally, in Section 4 we prove a central limit theorem for $Z(t)$ in the limit as $N \rightarrow+\infty$. 


\section{The model}

\subsection{Definition}

Consider a probability space in which a family $\{U(t, i) ; t, i \in \mathbb{N}\}$ of i.i.d. random variables with uniform distribution on $[0,1]$ is defined. For example, we take $\Omega=[0,1]^{\mathbb{N}^{2}}, \mathcal{F}=$ $\mathscr{B}([0,1])^{\otimes \mathbb{N}^{2}}$, and $\mathbb{P}=\lambda^{\otimes \mathbb{N}^{2}}$ (where $\mathcal{B}([0,1])$ and $\lambda$ respectively denote the Borel sigmaalgebra and the Lebesgue measure on $[0,1])$, with the $U(t, i)$ the canonical projections. We pose $\mathcal{F}_{t}=\sigma(U(s, i) ; 0 \leq s \leq t, i \in \mathbb{N})$.

Let $m=a+b$ be the total number of balls in a single urn at time 0 . In what follows we respectively denote by $X_{t}^{N}(i)$ and $Z_{t}^{N}(i)=X_{t}^{N}(i) /(t+m)$ the number and the fraction of red balls in urn $i$ at time $t$ for the model with $N$ urns. Then $X_{t}^{N}=\sum_{i=1}^{N} X_{t}^{N}(i)$ denotes the total number of red balls at time $t$ and

$$
Z_{t}^{N}=\frac{1}{N} \sum_{i=1}^{N} Z_{t}^{N}(i)=\frac{X_{t}}{N(t+m)}
$$

denotes be the total fraction of red balls at time $t$ in the model with $N$ urns.

Now, for a fixed $N \in \mathbb{N}$, we define, for each $i=1, \ldots, N$,

$$
\begin{aligned}
X_{0}^{N}(i) & =1, & Z_{0}^{N}(i) & =\frac{a}{m}, \\
X_{t+1}^{N}(i) & =X_{t}^{N}(i)+Y_{t}(i), & Z_{t+1}^{N}(i) & =\frac{t+m}{t+m+1} Z_{t}^{N}(i)+\frac{1}{t+m+1} Y_{t}(i),
\end{aligned}
$$

where $Y_{t}(i)=\mathbf{1}_{\left\{U(t+1, i) \leq \alpha Z_{t}^{N}+(1-\alpha) Z_{t}^{N}(i)\right\}}$.

A simple calculation shows that the random variables $Y_{t}(i)$ for $i=1, \ldots, N$ are conditionally independent given $\mathcal{F}_{t}$ and have conditional distribution which is Bernoulli with parameter $\alpha Z_{t}+(1-\alpha) Z_{t}(i)$.

\subsection{Basic properties}

Let $N \in \mathbb{N}$ be fixed. The following properties hold.

(P1) $Z^{N}=\left(Z_{t}^{N}\right)_{t \geq 0}$ is a bounded martingale.

Indeed,

$$
\begin{aligned}
\mathbb{E}\left[Z_{t+1}^{N} \mid \mathcal{F}_{t}\right] & =\mathbb{E}\left[\frac{t+m}{t+m+1} Z_{t}^{N}+\frac{1}{N(t+m+1)} \sum_{i=1}^{N} Y_{t}(i) \mid \mathcal{F}_{t}\right] \\
& =\frac{t+m}{t+m+1} Z_{t}^{N}+\frac{1}{t+m+1} \frac{1}{N} \sum_{i=1}^{N}\left[\alpha Z_{t}^{N}+(1-\alpha) Z_{t}(i)\right] \\
& =\frac{t+m}{t+m+1} Z_{t}^{N}+\frac{1}{t+m+1} Z_{t}^{N} \\
& =Z_{t}^{N}
\end{aligned}
$$

Consequently, $Z^{N}$ converges a.s. and in $L^{p}$ to a random variable which we denote by $Z_{\infty}^{N}$.

(P2) By symmetry reasons, for every fixed $t$, the random variables $Z_{t}^{N}(i)$ for $i=1, \ldots, N$ are exchangeable, and, thus, their mean value equals $a / m$. 
Indeed, since $Z^{N}$ is a martingale, for every $t$, we have

$$
\frac{a}{m}=\mathbb{E}\left[Z_{t}^{N}\right]=\mathbb{E}\left[\frac{1}{N} \sum_{i=1}^{N} Z_{t}^{N}(i)\right]=\frac{1}{N} \sum_{i=1}^{N} \mathbb{E}\left[Z_{t}^{N}(i)\right]=\mathbb{E}\left[Z_{t}^{N}(1)\right] .
$$

Note that we also have

$$
\mathbb{E}\left[Y_{t}(i)\right]=\mathbb{E}\left[\alpha Z_{t}^{N}+(1-\alpha) Z_{t}^{N}(i)\right]=\frac{a}{m} \quad \text { for all } t, i \in \mathbb{N} .
$$

(P3) $Z^{N}(i)=\left(Z_{t}^{N}(i)\right)_{t \geq 0}$ is a martingale if and only if $\alpha=0$.

Indeed,

$$
\begin{aligned}
\mathbb{E}\left[Z_{t+1}^{N}(i) \mid \mathcal{F}_{t}\right] & =\frac{t+m}{t+m+1} Z_{t}^{N}(i)+\frac{1}{t+m+1}\left[\alpha Z_{t}^{N}+(1-\alpha) Z_{t}^{N}(i)\right] \\
& =\frac{t+m+1-\alpha}{t+m+1} Z_{t}^{N}(i)+\frac{\alpha}{t+m+1} Z_{t}^{N} \\
& =Z_{t}^{N}(i) \text { for every } t
\end{aligned}
$$

if and only if $\alpha=0$ or $Z_{t}^{N}(i)=Z_{t}^{N}, \mathbb{P}$-a.s. for every $t$; but the last assertion is false, since, for example, when $t=1$, the random variables $Y_{0}(k)$ for $k=1, \ldots, N$ are i.i.d. Bernoulli of parameter $a / m$ and so $\mathbb{P}\left(Z_{1}^{N}(i)=Z_{1}^{N}\right)=\mathbb{P}\left(Y_{0}(i)=(1 / N) \sum_{k=1}^{N} Y_{0}(k)\right)<1$.

In the next section we study the behavior of $Z_{t}^{N}(i)$ for $i=1, \ldots, N$ when $t \rightarrow+\infty$. Since $N$ is fixed, we omit the superscript $N$ and write $Z_{t}$ and $Z_{t}(i)$ for $Z_{t}^{N}$ and $Z_{t}^{N}(i)$, respectively.

\section{Synchronization}

In this section we show that, as soon as $\alpha>0$, the urns synchronize a.s., i.e. for each $i=1, \ldots, N$,

$$
\lim _{t \rightarrow+\infty} Z_{t}(i)=\lim _{t \rightarrow+\infty} Z_{t} \text { a.s. }
$$

We proceeds in two steps. First we show $L^{2}$-synchronization, i.e.

$$
\lim _{t \rightarrow+\infty} \mathbb{E}\left[\left(Z_{t}(i)-Z_{t}\right)^{2}\right]=0 .
$$

Then, using bounds on the $L^{2}$ rate of convergence, we derive the almost-sure synchronization.

\section{1. $L^{2}$ synchronization}

In the following statement, for two positive sequences $a_{t}$ and $b_{t}$, we write $a_{t} \sim b_{t}$ if

$$
0<\liminf _{t \rightarrow+\infty} \frac{a_{t}}{b_{t}} \leq \limsup _{t \rightarrow+\infty} \frac{a_{t}}{b_{t}}<+\infty
$$

Theorem 1. The following asymptotic estimates hold:

$$
\mathbb{E}\left[\left(Z_{t}(i)-Z_{t}\right)^{2}\right] \sim \begin{cases}t^{-2 \alpha} & \text { for } 0<\alpha<\frac{1}{2}, \\ t^{-1} \log t & \text { for } \alpha=\frac{1}{2}, \\ t^{-1} & \text { for } \frac{1}{2}<\alpha \leq 1 .\end{cases}
$$

To prove Theorem 3.1, we need the following a technical lemma. 
Lemma 1. There exists a constant $C$ with $0<C<a / m^{2}$ such that, for every $t \geq 0$,

$$
\operatorname{var}\left(Z_{t}\right) \leq \frac{C}{N}
$$

In particular, we have

$$
\sup _{t} \mathbb{E}\left[Z_{t}^{2}\right]=\mathbb{E}\left[Z_{\infty}^{2}\right]<\frac{a}{m}
$$

Proof. Note that

$$
\operatorname{var}\left[Z_{t+1}\right]=\operatorname{var}\left[\mathbb{E}\left(Z_{t+1} \mid \mathcal{F}_{t}\right)\right]+\mathbb{E}\left[\operatorname{var}\left(Z_{t+1} \mid \mathcal{F}_{t}\right)\right]=\operatorname{var}\left[Z_{t}\right]+\mathbb{E}\left[\operatorname{var}\left(Z_{t+1} \mid \mathcal{F}_{t}\right)\right] .
$$

Moreover,

$$
\begin{aligned}
\mathbb{E}[\operatorname{var} & \left.\left(Z_{t+1} \mid \mathcal{F}_{t}\right)\right] \\
& =\mathbb{E}\left[\operatorname{var}\left(\frac{t+m}{t+m+1} Z_{t}+\frac{1}{(t+m+1) N} \sum_{i=1}^{N} Y_{t}(i) \mid \mathcal{F}_{t}\right)\right] \\
& =\mathbb{E}\left[\operatorname{var}\left(\frac{1}{(t+m+1) N} \sum_{i=1}^{N} Y_{t}(i) \mid \mathcal{F}_{t}\right)\right] \\
& =\mathbb{E}\left[\frac{1}{(t+m+1)^{2} N^{2}} \sum_{i=1}^{N} \operatorname{var}\left(Y_{t}(i) \mid \mathcal{F}_{t}\right)\right] \\
& =\mathbb{E}\left[\frac{1}{(t+m+1)^{2} N^{2}} \sum_{i=1}^{N}\left(\alpha Z_{t}+(1-\alpha) Z_{t}(i)\right)-\left(\alpha Z_{t}+(1-\alpha) Z_{t}(i)\right)^{2}\right] \\
& =\frac{1}{(t+m+1)^{2} N} \mathbb{E}\left[Z_{t}-\alpha^{2} Z_{t}^{2}-2\left(\alpha-\alpha^{2}\right) Z_{t}^{2}-\frac{(1-\alpha)^{2}}{N} \sum_{i=1}^{N} Z_{t}(i)^{2}\right] .
\end{aligned}
$$

Thus, the following recursive equation holds:

$$
\begin{aligned}
\operatorname{var}\left(Z_{t+1}\right)= & \operatorname{var}\left(Z_{t}\right)+\frac{1}{(t+m+1)^{2} N} \frac{a}{m}-\frac{\alpha(2-\alpha)}{(t+m+1)^{2} N}\left(\operatorname{var}\left(Z_{t}\right)+\frac{a^{2}}{m^{2}}\right) \\
& -\frac{(1-\alpha)^{2}}{(t+m+1)^{2} N^{2}} \sum_{i=1}^{N} \mathbb{E}\left[Z_{t}(i)^{2}\right] .
\end{aligned}
$$

In particular,

$$
\operatorname{var}\left(Z_{t+1}\right) \leq \operatorname{var}\left(Z_{t}\right)+\frac{1}{N(t+m+1)^{2}} \frac{a}{m},
$$

from which we obtain

$$
\operatorname{var}\left(Z_{t}\right) \leq \frac{a}{m} \frac{1}{N} \sum_{k=m+1}^{+\infty} \frac{1}{k^{2}}<\frac{a}{m^{2} N}
$$

Moreover,

$$
\mathbb{E}\left[Z_{t}^{2}\right]=\frac{a^{2}}{m^{2}}+\operatorname{var}\left(Z_{t}\right)<\frac{a^{2}+a}{m^{2}} \leq \frac{a}{m},
$$

where this last inequality is easily shown to be equivalent to $a \leq m-1$, which is clearly true. 
Finally, since $Z_{t}^{2}$ is a bounded submartingale,

$$
\sup _{t} \mathbb{E}\left[Z_{t}^{2}\right]=\lim _{t} \mathbb{E}\left[Z_{t}^{2}\right]=\mathbb{E}\left[Z_{\infty}^{2}\right] .
$$

Proof of Theorem 1. Setting

$$
x_{t}:=\mathbb{E}\left[\left(Z_{t}(i)-Z_{t}\right)^{2}\right]=\operatorname{var}\left(Z_{t}(i)-Z_{t}\right),
$$

we first obtain a recursive equation satisfied by $x_{t}$ :

$$
\begin{aligned}
& x_{t+1}=\mathbb{E}\left[\operatorname{var}\left(Z_{t+1}(i)-Z_{t+1} \mid \mathcal{F}_{t}\right)\right]+\operatorname{var}\left(\mathbb{E}\left[Z_{t+1}(i)-Z_{t+1} \mid \mathcal{F}_{t}\right]\right) \\
& =\mathbb{E}\left[\operatorname{var}\left(\frac{1}{t+m+1}\left\{(t+m)\left(Z_{t}(i)-Z_{t}\right)+Y_{t}(i)-\frac{1}{N} \sum_{i=1}^{N} Y_{t}(i)\right\} \mid \mathcal{F}_{t}\right)\right] \\
& +\operatorname{var}\left(\frac{t+m}{t+m+1} Z_{t}(i)+\frac{1}{t+m+1}\left[\alpha Z_{t}+(1-\alpha) Z_{t}(i)\right]-Z_{t}\right) \\
& =\frac{1}{(t+m+1)^{2}} \mathbb{E}\left[\operatorname{var}\left(Y_{t}(i)-\frac{1}{N} \sum_{j=1}^{N} Y_{t}(j) \mid \mathcal{F}_{t}\right)\right] \\
& +\operatorname{var}\left(\frac{t+m+1-\alpha}{t+m+1}\left[Z_{t}(i)-Z_{t}\right]\right) \\
& =\frac{1}{(t+m+1)^{2}}\left[\left(1-\frac{1}{N}\right)^{2}+\frac{N-1}{N^{2}}\right] \mathbb{E}\left[\operatorname{var}\left(Y_{t}(i) \mid \mathcal{F}_{t}\right)\right] \\
& +\left(\frac{t+m+1-\alpha}{t+m+1}\right)^{2} \operatorname{var}\left(Z_{t}-Z_{t}(i)\right) \\
& =\frac{1}{(t+m+1)^{2}} \frac{N-1}{N}\left(\frac{a}{m}-\mathbb{E}\left[\left(\alpha Z_{t}+(1-\alpha) Z_{t}(i)\right)^{2}\right]\right)+\left(1-\frac{\alpha}{t+m+1}\right)^{2} x_{t} \\
& =\frac{1}{(t+m+1)^{2}} \frac{N-1}{N}\left(\frac{a}{m}-\mathbb{E}\left[\left\{Z_{t}-(1-\alpha)\left(Z_{t}-Z_{t}(i)\right)\right\}^{2}\right]\right) \\
& +\left(1-\frac{\alpha}{t+m+1}\right)^{2} x_{t} \\
& =\frac{1}{(t+m+1)^{2}} \frac{N-1}{N}\left(\frac{a}{m}-(1-\alpha)^{2} x_{t}-\mathbb{E}\left[Z_{t}^{2}\right]\right)+x_{t}-\frac{2 \alpha}{(t+m+1)} x_{t} \\
& +\frac{\alpha^{2}}{(t+m+1)^{2}} x_{t} \text {. }
\end{aligned}
$$

In the last equality we used the fact that $\mathbb{E}\left[Z_{t}\left(Z_{t}-Z_{t}(i)\right)\right]=0$ since, by symmetry,

$$
\mathbb{E}\left[Z_{t}(i) Z_{t}\right]=\frac{1}{N} \sum_{j=1}^{N} \mathbb{E}\left[Z_{t}(j) Z_{t}\right]=\mathbb{E}\left[\left(\frac{1}{N} \sum_{j=1}^{N} Z_{t}(j)\right) Z_{t}\right]=\mathbb{E}\left[Z_{t}^{2}\right] .
$$

We have therefore obtained the recursive equation

$$
\begin{aligned}
x_{t+1}= & x_{t}-\frac{2 \alpha}{t+m+1} x_{t}+\frac{\alpha^{2}-(N-1)(1-\alpha)^{2} / N}{(t+m+1)^{2}} x_{t} \\
& +\frac{1}{(t+m+1)^{2}} \frac{N-1}{N}\left(\frac{a}{m}-\mathbb{E}\left[Z_{t}^{2}\right]\right) .
\end{aligned}
$$


Now set $A:=2 \alpha$ and $B:=\alpha^{2}-(N-1)(1-\alpha)^{2} / N$. Then

$$
f(t):=1-\frac{A}{t+m+1}+\frac{B}{(t+m+1)^{2}}, \quad g(t):=\frac{(N-1)\left(a / m-\mathbb{E}\left[Z_{t}^{2}\right]\right) / N}{(t+m+1)^{2}},
$$

so that

$$
x_{t+1}=f(t) x_{t}+g(t) .
$$

It is easily seen that $-1 \leq B \leq 1$ and, since Lemma 1 states that $a / m-\mathbb{E}\left[Z_{\infty}^{2}\right]>0$, we have

$$
0<\frac{(N-1)\left(a / m-\mathbb{E}\left[Z_{\infty}^{2}\right]\right) / N}{(t+m+1)^{2}} \leq g(t) \leq \frac{a / m}{(t+m+1)^{2}} .
$$

We also remark that $0<f(t)<1$ for every $t \geq 0$. To see this, observe first that

$$
f(0)=1-\frac{2 \alpha}{m+1}+\frac{\alpha^{2}-(N-1)(1-\alpha)^{2} / N}{(m+1)^{2}},
$$

as a function of $\alpha$, has a strictly negative derivative; being positive for $\alpha=1$, it must be positive for all $\alpha \in[0,1]$. Moreover,

$$
\begin{aligned}
\frac{\mathrm{d}}{\mathrm{d} t} f(t) & =\frac{2}{(t+m+1)^{3}}\left[\alpha(t+m+1)-\alpha^{2}+\frac{N-1}{N}(1-\alpha)^{2}\right] \\
& >\frac{2}{(t+m+1)^{3}}\left(\alpha-\alpha^{2}\right) \\
& \geq 0
\end{aligned}
$$

so $f(t)$ is increasing in $t$. Since $\lim _{t \rightarrow+\infty} f(t)=1$, we conclude that $0<f(t)<1$. Now set

$$
\xi_{t}:=\frac{x_{t}}{\prod_{k=0}^{t-1} f(k)} .
$$

By (2) we obtain

$$
\xi_{t+1}=\xi_{t}+F(t)
$$

where

$$
F(t):=\frac{g(t)}{\prod_{k=0}^{t} f(k)} .
$$

So, observing that $\xi_{0}=x_{0}=0$, we obtain

$$
\xi_{t}=\sum_{i=0}^{t-1} F(i)
$$

or, equivalently,

$$
x_{t}=\left[\prod_{k=0}^{t-1} f(k)\right] \sum_{i=0}^{t-1} F(i) .
$$


Now we can study the asymptotic behavior of each term. Note first that, as $t \rightarrow+\infty$,

$$
\begin{aligned}
\prod_{k=0}^{t-1} f(k) & =\exp \left[\sum_{k=0}^{t-1} \log \left(1-\frac{A}{k+m+1}+\frac{B}{(k+m+1)^{2}}\right)\right] \\
& =\exp \left[-2 \alpha \sum_{k=0}^{t-1} \frac{1}{k+m+1}+O(1)\right] \\
& =\exp [-2 \alpha \log (t+m)+O(1)] \\
& \sim t^{-2 \alpha}
\end{aligned}
$$

Since by (3) we have $g(t) \sim t^{-2}$,

$$
F(t) \sim t^{2 \alpha-2} \Longrightarrow \xi_{t}=\sum_{i=0}^{t-1} F(i) \sim \begin{cases}1 & \text { for } 0 \leq \alpha<\frac{1}{2} \\ \log t & \text { for } \alpha=\frac{1}{2} \\ t^{2 \alpha-1} & \text { for } \frac{1}{2}<\alpha \leq 1\end{cases}
$$

By (4), (5), and (6), the conclusion follows.

\subsection{Almost-sure synchronization}

For $\alpha=0$, the sequences $\left\{Z_{t}(i)\right\}_{t \geq 0}$ converge as $t \rightarrow+\infty$ to independent and betadistributed limits $Z_{\infty}(i)$. For $\alpha>0$, we have a quite different behavior.

Theorem 2. For each $i=1,2, \ldots, N$,

$$
\lim _{t \rightarrow+\infty} Z_{t}(i)=\lim _{t \rightarrow+\infty} Z_{t} \text { a.s. }
$$

Proof. By Theorem $1, Z_{t}(i)$ converges to $Z_{\infty}$ in $L^{2}$ as $t \mapsto \infty$. It is therefore enough to show that the almost-sure $\operatorname{limit}_{t \rightarrow+\infty} Z_{t}(i)$ exists. We observe that $\left\{Z_{t}(i)\right\}_{t}$ is a quasimartingale, i.e.

$$
\sum_{t=0}^{+\infty} \mathbb{E}\left[\left|\mathbb{E}\left[Z_{t+1}(i) \mid \mathcal{F}_{t}\right]-Z_{t}(i)\right|\right]<+\infty .
$$

Indeed, using the fact that

$$
\mathbb{E}\left[Z_{t+1}(i) \mid \mathcal{F}_{t}\right]=\left(1-\frac{\alpha}{t+m+1}\right) Z_{t}(i)+\frac{\alpha}{t+m+1} Z_{t},
$$

we obtain

$$
\begin{aligned}
\sum_{t} \mathbb{E}\left[\left|\mathbb{E}\left[Z_{t+1}(i) \mid \mathcal{F}_{t}\right]-Z_{t}(i)\right|\right] & =\sum_{t} \frac{\alpha}{t+m+1} \mathbb{E}\left[\left|Z_{t}(i)-Z_{t}\right|\right] \\
& \leq \sum_{t} \frac{\alpha}{t+m+1}\left(\mathbb{E}\left[\left(Z_{t}(i)-Z_{t}\right)^{2}\right]\right)^{1 / 2} \\
& <+\infty
\end{aligned}
$$

where the last inequality follows from Theorem 1 . The conclusion now follows from the fact that a bounded quasimartingale has an almost-sure limit (see, e.g. [10, p. 46]). 


\section{A central limit theorem}

In this section we study the limiting distribution of $Z_{t}$ in the limit as the number of urns, $N$, goes to $\infty$. To emphasize the dependence on the number of urns, we again use the notation $Z_{t}^{N}$ and set

$$
W_{t}^{N}:=\sqrt{N}\left(Z_{t}^{N}-\frac{a}{m}\right)
$$

Moreover, let $x_{t}^{\infty}$ be the solution of the recursion

$$
x_{t+1}^{\infty}=x_{t}^{\infty}-\frac{2 \alpha}{t+m+1} x_{t}^{\infty}+\frac{2 \alpha-1}{(t+m+1)^{2}} x_{t}^{\infty}+\frac{1}{(t+m+1)^{2}}\left(\frac{a}{m}-\frac{a^{2}}{m^{2}}\right),
$$

with $x_{0}^{\infty}=0$. Note that (7) is the limit as $N \rightarrow+\infty$ of (1), which implies that

$$
x_{t}^{\infty}=\lim _{N \rightarrow+\infty} \mathbb{E}\left[\left(Z_{t}^{N}(i)-Z_{t}^{N}\right)^{2}\right] .
$$

Theorem 3. The stochastic process $\left\{W_{t}^{N}: t \geq 0\right\}$ converges weakly as $N \rightarrow+\infty$ to the Gauss-Markov process solution of the recursion

$$
W_{t+1}=W_{t}+\sigma_{t} B_{t+1}, \quad W_{0}=\delta_{0},
$$

where

$$
\sigma_{t}^{2}=\frac{1}{(t+m+1)^{2}}\left(\left[\frac{a}{m}-\frac{a^{2}}{m^{2}}\right]-(1-\alpha)^{2} x_{t}^{\infty}\right) \geq 0
$$

and $\left\{B_{t}: t \geq 1\right\}$ is a sequence of i.i.d. $\mathcal{N}(0,1)$ random variables.

To prove Theorem 3, we require two technical lemmas.

Lemma 2. The following almost-sure limits hold for every $t \geq 1$ :

$$
\begin{gathered}
\lim _{N \rightarrow+\infty} Z_{t}^{N}=\frac{a}{m}, \\
\lim _{N \rightarrow+\infty} \frac{1}{N} \sum_{i=1}^{N}\left[Z_{t}^{N}(i)\right]^{2}=x_{t}^{\infty}+\frac{a^{2}}{m^{2}} .
\end{gathered}
$$

Proof. We first prove (10) by induction on $t$. Since $Z_{0}^{N} \equiv a / m$, there is nothing to prove for $t=0$. For simplicity, in the following formulae we use the earlier notation $Z_{t}$ and $Z_{t}(i)$, omitting the dependence on $N$. Recall that

$$
Z_{t+1}=Z_{t}+\frac{1}{t+m+1} \frac{1}{N} \sum_{i=1}^{N}\left[Y_{t}(i)-\alpha Z_{t}-(1-\alpha) Z_{t}(i)\right]
$$

Let

$$
V_{i}:=Y_{t}(i)-\alpha Z_{t}-(1-\alpha) Z_{t}(i) .
$$

By assumption, the $V_{i}$ are independent for the conditional probability $\mathbb{P}\left(\cdot \mid \mathcal{F}_{t}\right)$ and $\mathbb{E}\left[V_{i} \mid \mathcal{F}_{t}\right]=$ 0 . Since the $V_{i}$ are bounded, a strong law of large numbers holds: letting

$$
F:=\left\{\lim _{N \rightarrow+\infty} \frac{1}{N} \sum_{i=1}^{N} V_{i}=0\right\}
$$


we have $\mathbb{P}\left(F \mid \mathcal{F}_{t}\right)=1$ a.s., which implies that $\mathbb{P}(F)=1$. Thus, by (12),

$$
\lim _{N \rightarrow+\infty} Z_{t+1}=\lim _{N \rightarrow+\infty} Z_{t}=\frac{a}{m} \quad \text { a.s., }
$$

where we have used the inductive assumption.

We now prove (11), again by induction on $t$. Using

$$
Z_{t+1}(i)=\frac{t+m}{t+m+1} Z_{t}(i)+\frac{1}{t+m+1} Y_{t}(i),
$$

we obtain

$$
\begin{aligned}
Z_{t+1}^{2}(i)= & \left(\frac{t+m}{t+m+1}\right)^{2} Z_{t}^{2}(i)+\frac{1}{(t+m+1)^{2}} Y_{t}(i)+\frac{2(t+m)}{(t+m+1)^{2}} Z_{t}(i) Y_{t}(i) \\
= & \left(\frac{t+m}{t+m+1}\right)^{2} Z_{t}^{2}(i)+\frac{1}{(t+m+1)^{2}} V_{i}+\frac{\alpha Z_{t}+(1-\alpha) Z_{t}(i)}{(t+m+1)^{2}} \\
& +\frac{2(t+m)}{(t+m+1)^{2}} Z_{t}(i) V_{i}+\frac{2(t+m)}{(t+m+1)^{2}} Z_{t}(i)\left[\alpha Z_{t}+(1-\alpha) Z_{t}(i)\right]
\end{aligned}
$$

which gives

$$
\begin{aligned}
\frac{1}{N} \sum_{i=1}^{N} Z_{t+1}^{2}(i)= & \frac{(t+m)(t+m+2-2 \alpha)}{(t+m+1)^{2}} \frac{1}{N} \sum_{i=1}^{N} Z_{t}^{2}(i)+\frac{1}{(t+m+1)^{2}} Z_{t} \\
& +\frac{2 \alpha(t+m)}{(t+m+1)^{2}} Z_{t}^{2}+\frac{1}{(t+m+1)^{2}} \frac{1}{N} \sum_{i=1}^{N} V_{i} \\
& +\frac{2(t+m)}{(t+m+1)^{2}} \frac{1}{N} \sum_{i=1}^{N} Z_{t}(i) V_{i} .
\end{aligned}
$$

The fact that $(1 / N) \sum_{i=1}^{N} Z_{t+1}^{2}(i)$ converges a.s., as $N$ tends to $\infty$, to a constant follows from (13) using

- the inductive assumption;

- the fact, already proved, that $\lim _{N \rightarrow \infty} Z_{t}=a / m$ a.s.;

- the fact that

$$
\frac{1}{N} \sum_{i=1}^{N} V_{i} \rightarrow 0 \quad \text { a.s. }
$$

as shown in the proof of (10); and

- the fact that

$$
\frac{1}{N} \sum_{i=1}^{N} Z_{t}(i) V_{i} \rightarrow 0 \quad \text { a.s. }
$$

which is proved in the same way. 
To identify this limit, note that

$$
\frac{1}{N} \sum_{i=1}^{N} \mathbb{E}\left[Z_{t}(i)^{2}\right]=\operatorname{var}\left(Z_{t}-Z_{t}(i)\right)+\mathbb{E}\left[Z_{t}^{2}\right]=x_{t}+\operatorname{var}\left(Z_{t}\right)+\frac{a^{2}}{m^{2}} .
$$

By Lemma $1, \operatorname{var}\left(Z_{t}\right)$ tends to 0 as $N \rightarrow+\infty$. Finally, since $x_{t}$ satisfies (1), it is easily proved by induction on $t$ that $x_{t}$ has a limit $x_{t}^{\infty}$ as $N \rightarrow+\infty$, and this limit satisfies (7).

Remark 1. From Lemma 2, it follows that $\sigma_{t}^{2} \geq 0$, where $\sigma_{t}$ has been defined in (9). Indeed,

$$
\frac{a}{m}=\lim _{N \rightarrow+\infty} \frac{1}{N} \sum_{i=1}^{N} Z_{t}^{N}(i) \geq \lim _{N \rightarrow+\infty} \frac{1}{N} \sum_{i=1}^{N}\left[Z_{t}^{N}(i)\right]^{2}=x_{t}^{\infty}+\frac{a^{2}}{m^{2}} ;
$$

thus,

which implies that $\sigma_{t}^{2} \geq 0$.

$$
x_{t}^{\infty} \leq \frac{a}{m}-\frac{a^{2}}{m^{2}}
$$

Lemma 3. It holds that

$$
\mathbb{E}\left[\mathrm{e}^{\mathrm{i} u W_{t+1}^{N}} \mid \mathscr{F}_{t}\right]=\mathrm{e}^{\mathrm{i} u W_{t}^{N}} \exp \left[-\frac{u^{2}}{2} \frac{1}{(t+m+1)^{2}}\left[\left(\frac{a}{m}-\frac{a^{2}}{m^{2}}\right)-(1-\alpha)^{2} x_{t}^{\infty}+\varepsilon_{N}\right]\right],
$$

where $\varepsilon_{N}$ is a sequence of $\mathscr{F}_{t}$-measurable, uniformly bounded random variables such that $\varepsilon_{N} \rightarrow 0$ a.s.

Proof. Using the recursive equation

$$
W_{t+1}^{N}=\frac{t+m}{t+m+1} W_{t}^{N}+\frac{1}{t+m+1}\left(\frac{1}{\sqrt{N}} \sum_{i=1}^{N} Y_{t}(i)-\frac{a}{m} \sqrt{N}\right)
$$

and using the notation $s=m+t$, we have

$$
\begin{aligned}
\mathbb{E}\left[\mathrm{e}^{\mathrm{i} u W_{t+1}^{N}} \mid \mathcal{F}_{t}\right]= & \exp \left[\mathrm{i} u \frac{s}{s+1} W_{t}^{N}\right] \exp \left[-\mathrm{i} \frac{u}{s+1} \frac{a \sqrt{N}}{m}\right] \\
& \times \mathbb{E}\left[\prod_{j=1}^{N} \exp \left[\mathrm{i} \frac{u}{\sqrt{N}(s+1)} Y_{t}(j)\right] \mid \mathcal{F}_{t}\right] .
\end{aligned}
$$

Compute, using the characteristic function of a Bernoulli distribution,

$$
\begin{aligned}
\mathbb{E} & {\left[\prod_{j=1}^{N} \exp \left[\mathrm{i} \frac{u}{\sqrt{N}(s+1)} Y_{t}(j)\right] \mid \mathcal{F}_{t}\right] } \\
& =\prod_{j=1}^{N}\left[1+\left(\exp \left[\mathrm{i} \frac{u}{\sqrt{N}(s+1)}\right]-1\right)\left(\alpha Z_{t}+(1-\alpha) Z_{t}(j)\right)\right] \\
& =\exp \left[\sum_{j=1}^{N} \log \left(1+\left(\frac{i u}{\sqrt{N}(s+1)}-\frac{u^{2}}{2 N(s+1)^{2}}+o\left(\frac{1}{N}\right)\right)\left(\alpha Z_{t}+(1-\alpha) Z_{t}(j)\right)\right)\right] \\
& =\exp \left[\frac{i u \sqrt{N}}{s+1} Z_{t}-\frac{u^{2}}{2(s+1)^{2}} Z_{t}+\frac{u^{2}}{2(s+1)^{2}} \frac{1}{N} \sum_{j=1}^{N}\left(\alpha Z_{t}+(1-\alpha) Z_{t}(j)\right)^{2}+o(1)\right]
\end{aligned}
$$


where the $o$ (1) term on the right-hand side denotes a uniformly bounded sequence of random variables that tends to 0 a.s. as $N \rightarrow+\infty$, which, in what follows, may change from line to line.

Recalling that $Z_{t}=W_{t}^{(N)} / \sqrt{N}+a / m$, the first term in the argument of the exponential above equals

$$
\mathrm{i} u \frac{1}{s+1} W_{t}+\mathrm{i} \frac{u}{s+1} \frac{a \sqrt{N}}{m} .
$$

Thus,

$$
\begin{aligned}
\mathbb{E}\left[\mathrm{e}^{\mathrm{i} u W_{t+1}^{N}} \mid \mathcal{F}_{t}\right]=\mathrm{e}^{\mathrm{i} u W_{t}^{N}} \exp \left[-\frac{u^{2}}{2(s+1)^{2}}\left(Z_{t}-\alpha^{2} Z_{t}^{2}-2 \alpha(1-\alpha) Z_{t}^{2}\right.\right. & \\
& \left.\left.-(1-\alpha)^{2} \frac{1}{N} \sum_{j=1}^{N} Z_{t}^{2}(j)\right)+o(1)\right],
\end{aligned}
$$

which, using Lemma 2, yields

$$
\mathbb{E}\left[\mathrm{e}^{\mathrm{i} u W_{t+1}^{N}} \mid \mathscr{F}_{t}\right]=\mathrm{e}^{\mathrm{i} u W_{t}^{N}} \exp \left[-\frac{u^{2}}{2} \frac{1}{(s+1)^{2}}\left(\frac{a}{m}-\frac{a^{2}}{m^{2}}-(1-\alpha)^{2} x_{t}^{\infty}\right)+o(1)\right] .
$$

Proof of Theorem 3. Since

$$
\mathbb{E}\left[\exp \left[\mathrm{i} \sum_{r=1}^{t+1} u_{r} W_{r}^{N}\right]\right]=\mathbb{E}\left[\exp \left[\mathrm{i} \sum_{r=1}^{t} u_{r} W_{r}^{N}\right] \mathbb{E}\left[\exp \left[\mathrm{i} u_{t+1} W_{t+1}^{N}\right] \mid \mathcal{F}_{t}\right]\right]
$$

by Lemma 3, we easily see by induction on $t$ that the limits

$$
\varphi_{t}\left(u_{1}, \ldots, u_{t}\right):=\lim _{N \rightarrow \infty} \mathbb{E}\left[\exp \left[\mathrm{i} \sum_{r=1}^{t} u_{r} W_{r}^{N}\right]\right]
$$

exist, and satisfy the recursion

$$
\begin{aligned}
& \varphi_{t+1}\left(u_{1}, \ldots, u_{t-1}, u_{t}, u_{t+1}\right) \\
& \quad=\varphi_{t}\left(u_{1}, \ldots, u_{t-1}, u_{t}+u_{t+1}\right) \exp \left[-\frac{u^{2}}{2(t+m+1)^{2}}\left(\frac{a}{m}-\frac{a^{2}}{m^{2}}-(1-\alpha)^{2} x_{t}^{\infty}\right)\right],
\end{aligned}
$$

with initial condition $\varphi_{0}=1$. It is now straightforward to check that the Gaussian process in (8) gives rise to the same recursion for the characteristic functions.

Remark 2. Note that, for finite $N,\left(W_{t}^{N}\right)$ is not a Markov process: we need the whole vector $\left(Z_{t}(i)\right)_{i=1}^{N}$ to have a Markov process. The Markov property is recovered in the limit as $N \rightarrow+\infty$.

\section{References}

[1] ACEBRón, J. et al. (2005). The Kuramoto model: a simple paradigm for synchronization phenomena. Rev. Modern Phys. 77, 137.

[2] Berglund, N., Fernandez, B. and Gentz, B. (2007). Metastability in interacting nonlinear stochastic differential equations. I. From weak coupling to synchronization. Nonlinearity 20, 2551-2581.

[3] Bovier, A. (2006). Metastability: a potential theoretic approach. In International Congress of Mathematicians, Vol. III, European Mathenatical Society, Zürich, pp. 499-518. 
[4] Cirillo, P., Gallegati, M. and Hüsler, J. (2012). A Pólya lattice model to study leverage dynamics and contagious financial fragility. Adv. Complex Systems 15.

[5] Klenke, A. (2008). Probability Theory. A Comprehensive Course. Springer, London.

[6] Kuramoto, Y. (2003). Chemical Oscillations, Waves, and Turbulence. Dover, Mineola, NY.

[7] Launay, M. (2011). Interacting urn models. Preprint. Available at http://arxiv.org/abs/1101.1410.

[8] Launay, M. And Limic, V. (2012). Generalized interacting urn models. Preprint. Available at http://arxiv.org/abs/ 1207.5635 .

[9] Marsili, M. and Valleriani, A. (1998). Self organization of interacting Pólya urns. Europ. Physical J. B 3, 417-420.

[10] MÉtivier, M. (1982). Semimartingales (de Gruyter Studies Math. 2). De Gruyter, Berlin.

[11] Paganoni, A. M. And Secchi, P. (2004). Interacting reinforced-urn systems. Adv. Appl. Prob. 36, $791-804$.

[12] Pemantle, R. (2007). A survey of random processes with reinforcement. Prob. Surveys 4, 1-79.

[13] Prindle, A. et al. (2011). A sensing array of radically coupled genetic 'biopixels'. Nature 481, 39-44. 